\title{
Differential Evolution: An Inverse Approach for Crack Detection
}

\author{
Prabir Kumar Jena, ${ }^{1}$ Dhirendra Nath Thatoi, ${ }^{2}$ and Dayal R. Parhi ${ }^{1}$ \\ ${ }^{1}$ Department of Mechanical Engineering, NIT Rourkela, Odisha 769008, India \\ ${ }^{2}$ Department of Mechanical Engineering, Siksha O Anusandhan Deemed University, Bhubaneswar, Odisha 751030, India
}

Correspondence should be addressed to Dhirendra Nath Thatoi; dnthatoi@gmail.com

Received 30 April 2013; Revised 2 September 2013; Accepted 13 September 2013

Academic Editor: Marc Thomas

Copyright (C) 2013 Prabir Kumar Jena et al. This is an open access article distributed under the Creative Commons Attribution License, which permits unrestricted use, distribution, and reproduction in any medium, provided the original work is properly cited.

\begin{abstract}
This paper presents a damage detection technique combining analytical and experimental investigations on a cantilever aluminium alloy beam with a transverse surface crack. Firstly, the first three natural frequencies were determined using analytical methods based on strain energy release rate. Secondly, an experimental method was adopted to validate the theoretical findings. The damage location and severity assessment is the third stage and is formulated as a constrained optimisation problem and solved using the proposed differential evolution (DE) algorithm based on the measured and calculated first three natural frequencies as inputs. Numerical simulation studies indicate that the proposed method is robust and can be used effectively in structural health monitoring (SHM) applications.
\end{abstract}

\section{Introduction}

A crack is a potential source of catastrophic failure in structures. Extensive investigations by researchers have been done to develop structural integrity monitoring techniques. Vibration measurement and analysis being an effective and convenient way to detect cracks in structures is mostly being used for development of various such techniques.

Several nondestructive techniques (NDT) are available for local damage detection [1] using experimental methods like radiography, the magnetic field method, the acoustic method, and so forth. However, for health monitoring of critical and complex structures, in such experimental methods which require prior knowledge of the damage vicinity, accurate predictions may not be suitable. This has led to the development of quantitative global damage detection methods which are based on modal analysis [2, 3]. Researchers [4] also argue that in view of prohibitive costs and efforts involved in predicting damage to a high level accuracy a better idea is to roughly locate damage in the structure and then use standard NDT methods for closer analysis of the damaged area.

Recently a lot of work has been done using modal analysis to detect, locate, and predict crack severity to a greater accuracy level. Dimarogonas [2] in 1996 provided a comprehensive review of vibration based mode shape analysis followed by some recent reviews [4-7]. The damage detection problem can be defined as a nonlinear inverse problem [3]. In conventional model-based detection methods, the minimization of an objective function is defined in terms of the differences between the vibration data obtained by modal testing and those computed from the analytical model. These conventional optimization methods are gradient based and usually lead to a local minimum only. For a more accurate and reliable solution, global optimization techniques are developed such as genetic algorithms (GAs) $[8,9]$, artificial neural networks (ANNs) $[10,11]$, fuzzy logic $[12,13]$, particle swarm optimization (PSO) [14-19], and some hybrid methods like genetic fuzzy systems [20]. All these approaches have their strengths and weaknesses. However, there is a growing preference for the soft computing approaches based on dfferential evolution (DE) [21,22] due to faster and accurate predictions of global optimization to the objective function. A differential quadrature method combined with an evolutionary optimization algorithm has been proposed for crack detection in cylindrical shell structures. The circumferential crack, which is assumed to be open, is modeled by the extended rotational spring. For acceptability of this 


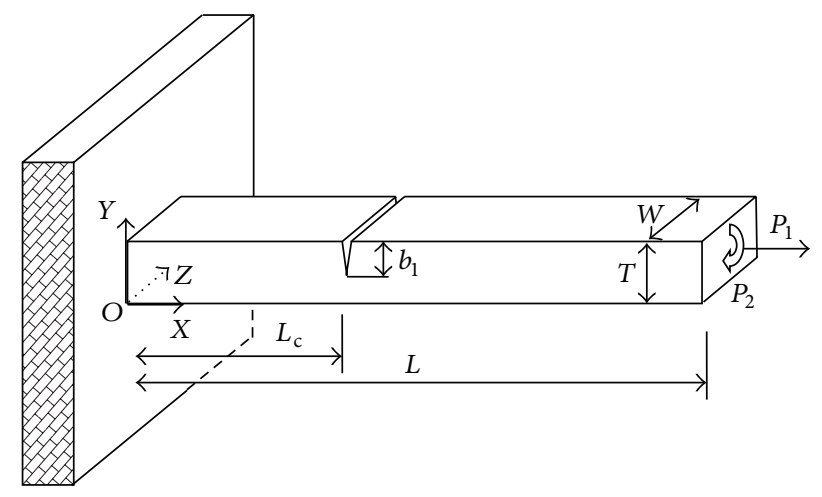

Figure 1: Beam model.

method an experimental analysis is also carried out [23]. A comparison has been made between the coupled local minimizers (CLM) method and the differential evolution (DE) algorithm to perform FE model updating for the damage detection in a cracked beam. CLM method is a gradient-based method with multiple local optimization runs whereas the DE algorithm is a direct search approach which uses a population of solution vectors collecting the design parameters [24].

In the current work, a systematic procedure has been developed to calculate the natural frequencies and mode shapes of a cracked cantilever beam with a transverse crack. The process endeavours to study the influence of a crack on natural frequencies and mode shapes. For different relative crack depths and locations, natural frequencies and the mode shapes of the cracked cantilever beam specimen have been found out using a theoretical method.

The rest of this paper is organized as follows. Section 2 introduces the theoretical analysis of a cracked beam structure. The experimental results are presented in Section 3, followed by Section 4 which includes the proposed DE algorithm. The results and discussions as well as conclusions are discussed in Sections 5 and 6, respectively.

\section{Mathematical Formulation}

2.1. Computation of Flexibility Matrix of a Damaged Beam Subjected to Complex Loading. A beam with cracks has smaller stiffness than that of a normal beam. This decreased local stiffness can be formulated as a matrix. The dimension of the matrix would depend on the degrees of freedom in the problem. Figure 1 shows a cantilever beam of width $W$ and height $T$, having a transverse surface crack of depth $b_{1}$. The beam experiences combined longitudinal and transverse motion due to the axial force $P_{1}$ and bending moment $P_{2}$. Here we consider two degrees of freedom, leading to a $2 \times$ 2 local stiffness matrix.

The relationship between strain energy release rate $J(b)$ and stress intensity factors $\left(G_{I i}, i=1\right.$ to 2$)$ at the crack section is given by Tada et al. [25] as

$$
J(b)=\frac{1}{E^{\prime}}\left(G_{I 1}+G_{I 2}\right)^{2},
$$

where $E^{\prime}=E /\left(1-v^{2}\right)$, for plane strain condition, $E^{\prime}=E$, for plane stress condition,

$G_{I 1}=$ stress intensity factor for opening mode I due to load $P_{1}$,

$G_{I 2}=$ stress intensity factor for opening mode I due to load $P_{2}$.

From earlier studies [25], the values of stress intensity factors are

$$
G_{I 1}=\frac{P_{1}}{W T} \sqrt{\pi b}\left(F_{1}\left(\frac{b}{t}\right)\right), \quad G_{I 2}=\frac{6 P_{2}}{W T^{2}} \sqrt{\pi b}\left(F_{2}\left(\frac{b}{t}\right)\right),
$$

$$
0 \leq b \leq b_{1}
$$

where the experimentally determined functions $F_{1}$ and $F_{2}$ are expressed as follows:

$$
\begin{aligned}
& F_{1}\left(\frac{b}{T}\right) \\
& =\left(\frac{2 T}{\pi b} \tan \left(\frac{\pi b}{2 T}\right)\right)^{0.5} \\
& \quad \times\left\{\frac{0.752+2.02(b / T)+0.37(1-\sin (\pi b / 2 T))^{3}}{\cos (\pi b / 2 T)}\right\}
\end{aligned}
$$

$$
\begin{aligned}
F_{2}\left(\frac{b}{T}\right)= & \left(\frac{2 T}{\pi b} \tan \left(\frac{\pi b}{2 T}\right)\right)^{0.5} \\
& \times\left\{\frac{0.923+0.199(1-\sin (\pi b / 2 T))^{4}}{\cos (\pi b / 2 T)}\right\} .
\end{aligned}
$$

The strain energy release rate (also called strain energy density function) at the crack location is defined as $J(b)=$ $\partial U_{t} / \partial(b \times W)$, where $(b \times W)$ is the newly created surface area of the crack.

$\Rightarrow d U_{t}=J(b) d(b \times W)=W J(b) d b$, since the width of the cross-section of the beam is constant.

So the strain energy release $\left(U_{t}\right)$ due to the crack of depth $b_{1}$ is calculated as

$$
U_{t}=W \int_{0}^{b_{1}} J(b) d b
$$

Then from Castigliano's theorem, the additional displacement along the force $P_{i}$ is

$$
S_{i}=\frac{\partial U_{t}}{\partial P_{i}}
$$

From (1) and (3), thus we have

$$
S_{i}=\frac{\partial}{\partial P_{i}}\left[W \int_{0}^{b_{1}} J(b) d b\right]=W \frac{\partial}{\partial P_{i}}\left[\int_{0}^{b_{1}} J(b) d b\right] .
$$



tion

The flexibility influence coefficient $C_{i j}$ will be by defini-

$$
\begin{aligned}
C_{i j} & =\frac{\partial S_{i}}{\partial P_{j}}=\frac{\partial}{\partial P_{j}}\left[W \frac{\partial}{\partial P_{i}}\left\{\int_{0}^{b_{1}} J(b) d b\right\}\right] \\
& =W \frac{\partial^{2}}{\partial P_{i} \partial P_{j}} \int_{0}^{b_{1}} J(b) d b .
\end{aligned}
$$

Substituting (1) in (6), we have

$$
C_{i j}=\frac{W}{E^{\prime}} \frac{\partial^{2}}{\partial P_{i} \partial P_{j}} \int_{0}^{b_{1}}\left(G_{l 1}+G_{l 2}\right)^{2} d b .
$$

Using $\delta=(b / T), d \delta=d b / T$, the following expressions are found out $d b=T d \delta$, and, when $b=0, \delta=0 ; b=b_{1}, \delta=$ $b_{1} / T=\delta_{1}$.

From the above condition, (9) can be written as

$$
C_{i j}=\frac{W T}{E^{\prime}} \frac{\partial^{2}}{\partial P_{i} \partial P_{j}} \int_{0}^{\delta_{1}}\left(G_{l 1}+G_{l 2}\right)^{2} d \delta
$$

where $\mathrm{C}_{i j}=$ flexibility influence coefficient in $i$ direction ( $x$-direction or $y$-direction) due to the load in $j$ direction $\left(P_{1}\right.$ or $\left.P_{2}\right)$.

Calculating $C_{11}, C_{12}\left(=C_{21}\right)$, and $C_{22}$ is as follows:

$$
\begin{gathered}
C_{11}=\frac{W T}{E^{\prime}} \int_{0}^{\delta_{1}} \frac{\pi b}{W^{2} T^{2}} 2 F_{1}^{2}(\delta) d \delta \\
=\frac{2 \pi}{W E^{\prime}} \int_{0}^{\delta_{1}} \delta F_{1}^{2}(\delta) d \delta \\
C_{12}=C_{21}=\frac{12 \pi}{E^{\prime} T W} \int_{0}^{\delta_{1}} \delta F_{1}(\delta) F_{2}(\delta) d \delta, \\
C_{22}=\frac{72 \pi}{E^{\prime} W T^{2}} \int_{0}^{\delta_{1}} \delta F_{2}^{2}(\delta) d \delta .
\end{gathered}
$$

The local stiffness matrix can be obtained using the inverse of compliance matrix

$$
K=\left[\begin{array}{ll}
K_{11} & K_{12} \\
K_{21} & K_{22}
\end{array}\right]=\left[\begin{array}{ll}
C_{11} & C_{12} \\
C_{21} & C_{22}
\end{array}\right]^{-1} .
$$

Converting the influence coefficient into dimensionless form we get

$$
\begin{aligned}
& \overline{C_{11}}=C_{11} \frac{W E^{\prime}}{2 \pi}, \\
& \overline{C_{12}}=C_{12} \frac{E^{\prime} T W}{12 \pi}=\overline{C_{21}}, \\
& \overline{C_{22}}=C_{22} \frac{E^{\prime} W T^{2}}{72 \pi} .
\end{aligned}
$$

2.2. Governing Equations for Vibration Mode of the Cracked Beam. The cantilever beam as mentioned in Section 2.1 is being considered for free vibration analysis. A cantilever

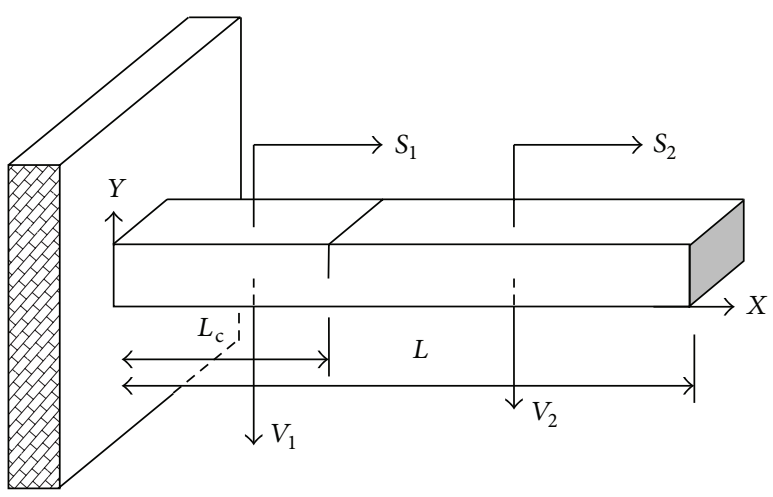

FIGURE 2: Beam model with deflections.

beam of length " $L$ ", width " $W$," and depth " $T$ " with a crack of depth " $b_{1}$ " at a distance " $L_{c}$ " from the fixed end is considered as shown in Figure 1. Considering $S_{1}(x, t)$ and $S_{2}(x, t)$ as the amplitudes of longitudinal vibration for the sections before and after the crack position and $V_{1}(x, t)$ and $V_{2}(x, t)$ as the amplitudes of bending vibration for the same section as shown in Figure 2, the free vibration of an Euler-Bernoulli beam of a constant rectangular cross-section is given by the following differential equations:

longitudinal vibration

$$
\frac{\partial^{2} S}{\partial t^{2}}=\left(\frac{E}{\rho}\right) \frac{\partial^{2} S}{\partial x^{2}}
$$

lateral vibration

$$
E I \frac{\partial^{4} V}{\partial x^{4}}-\rho \omega^{2} V=0 .
$$

The normal functions for the cracked beam in nondimensional form for both the longitudinal and bending vibrations in steady state can be defined as

$$
\begin{array}{r}
\bar{S}_{1}(\bar{x})=B_{1} \cos \left(\bar{H}_{s} \bar{x}\right)+B_{2} \sin \left(\bar{H}_{s} \bar{x}\right), \\
\bar{S}_{2}(\bar{x})=B_{3} \cos \left(\overline{H_{s}} \bar{x}\right)+B_{4} \sin \left(\overline{H_{s}} \bar{x}\right), \\
\bar{V}_{1}(\bar{x})=B_{5} \cosh \left(\overline{H_{v}} \bar{x}\right)+B_{6} \sinh \left(\overline{H_{v}} \bar{x}\right) \\
+B_{7} \cos \left(\overline{H_{v}} \bar{x}\right)+B_{8} \sin \left(\overline{H_{v}} \bar{x}\right), \\
\overline{V_{2}}(\bar{x})=B_{9} \cosh \left(\overline{H_{v}} \bar{x}\right)+B_{10} \sinh \left(\overline{H_{v}} \bar{x}\right) \\
+B_{11} \cos \left(\overline{H_{v}} \bar{x}\right)+B_{12} \sin \left(\overline{H_{v}} \bar{x}\right),
\end{array}
$$


where

$$
\begin{gathered}
\bar{x}=\frac{x}{L}, \quad \bar{S}=\frac{S}{L}, \quad \bar{V}=\frac{V}{L}, \quad \alpha=\frac{L_{c}}{L}, \\
\overline{H_{s}}=\frac{\omega L}{D_{s}}, \quad D_{s}=\left(\frac{E}{\rho}\right)^{1 / 2}, \quad \overline{H_{V}}=\left(\frac{\omega L^{2}}{D_{v}}\right)^{1 / 2}, \\
D_{v}=\left(\frac{E I}{\mu}\right)^{1 / 2}, \quad \mu=A \rho .
\end{gathered}
$$

$B_{i}(i=1,12)$ are the constants to be determined, from boundary conditions. The boundary conditions of the cantilever beam considered in the present study are

$$
\begin{aligned}
& \overline{S_{1}}(0)=0, \quad \overline{V_{1}}(0)=0, \quad{\overline{V_{1}}}^{\prime}(0)=0, \\
& \bar{S}_{2}^{\prime}(1)=0, \quad{\overline{V_{2}}}^{\prime \prime}(1)=0, \quad{\overline{V_{2}}}^{\prime \prime \prime}(1)=0 \text {. }
\end{aligned}
$$

At the cracked section

$$
\begin{aligned}
& \bar{S}_{1}(\alpha)=\bar{S}_{2}(\alpha), \quad \bar{V}_{1}(\alpha)=\bar{V}_{2}(\alpha), \\
& {\overline{V_{1}}}^{\prime \prime}(\alpha)={\overline{V_{2}}}^{\prime \prime}(\alpha), \quad{\overline{V_{1}}}^{\prime \prime \prime}(\alpha)={\overline{V_{2}}}^{\prime \prime \prime}(\alpha) .
\end{aligned}
$$

Also at the cracked section, we have

$$
\begin{aligned}
A E \frac{d S_{1}\left(L_{c}\right)}{d x}= & K_{11}\left(S_{2}\left(L_{c}\right)-S_{1}\left(L_{c}\right)\right) \\
& +K_{12}\left(\frac{d V_{2}\left(L_{c}\right)}{d x}-\frac{d V_{1}\left(L_{c}\right)}{d x}\right) .
\end{aligned}
$$

Multiplying both sides of the above equation by $A E / L K_{11} K_{12}$ we get

$$
\begin{aligned}
N_{1} N_{2}{\overline{S_{1}}}^{\prime}(\alpha)= & N_{2}\left(\overline{S_{2}}(\alpha)-\overline{S_{1}}(\alpha)\right) \\
& +N_{1}\left({\overline{V_{2}}}^{\prime}(\alpha)-{\overline{V_{1}}}^{\prime}(\alpha)\right) .
\end{aligned}
$$

Similarly,

$$
\begin{aligned}
E I \frac{d^{2} V_{1}\left(L_{c}\right)}{d x^{2}}= & K_{21}\left(S_{2}\left(L_{c}\right)-S_{1}\left(L_{c}\right)\right) \\
& +K_{22}\left(\frac{d V_{2}\left(L_{c}\right)}{d x}-\frac{d V_{1}\left(L_{c}\right)}{d x}\right) .
\end{aligned}
$$

Multiplying both sides of the aforementaioned equation by EI $/ L^{2} K_{22} K_{21}$ we get

$$
\begin{aligned}
N_{3} N_{4}{\overline{V_{1}}}^{\prime \prime}(\alpha)= & N_{3}\left(\overline{S_{2}}(\alpha)-\overline{S_{1}}(\alpha)\right) \\
& +N_{4}\left({\overline{V_{2}}}^{\prime}(\alpha)-{\overline{V_{1}}}^{\prime}(\alpha)\right),
\end{aligned}
$$

where

$$
\begin{array}{ll}
N_{1}=\frac{A E}{L K_{11}}, & N_{2}=\frac{A E}{K_{12}}, \\
N_{3}=\frac{E I}{L K_{22}}, & N_{4}=\frac{E I}{L^{2} K_{21}} .
\end{array}
$$

The normal functions ((16a)-(16d)) along with the boundary conditions as mentioned above ((18a) to (23)) yield the characteristic equation of the system as

$$
|Q|=0 \text {, }
$$

where $Q$ is a $12 \times 12$ matrix as given below, whose determinant is a function of natural circular frequency $(\omega)$, the relative location of the crack $(\alpha)$, and the local stiffness matrix $(K)$, which in turn is a function of the relative crack depth $\delta_{1}=$ $\left(b_{1} / T\right)$ :

$$
Q=\left[\begin{array}{cccccccccccc}
1 & 0 & 1 & 0 & 0 & 0 & 0 & 0 & 0 & 0 & 0 & 0 \\
0 & 1 & 0 & 1 & 0 & 0 & 0 & 0 & 0 & 0 & 0 & 0 \\
0 & 0 & 0 & 0 & G_{3} & G_{4} & -G_{7} & -G_{8} & 0 & 0 & 0 & 0 \\
0 & 0 & 0 & 0 & G_{4} & G_{3} & G_{8} & -G_{7} & 0 & 0 & 0 & 0 \\
G_{1} & G_{2} & -G_{5} & -G_{6} & -G_{1} & -G_{2} & G_{5} & G_{6} & 0 & 0 & 0 & 0 \\
G_{2} & G_{1} & G_{6} & -G_{5} & -G_{2} & -G_{1} & -G_{6} & G_{5} & 0 & 0 & 0 & 0 \\
G_{1} & G_{2} & G_{5} & G_{6} & -G_{1} & -G_{2} & -G_{5} & -G_{6} & 0 & 0 & 0 & 0 \\
S_{1} & S_{2} & S_{3} & S_{4} & -G_{2} & -G_{1} & G_{6} & -G_{5} & S_{5} & S_{6} & S_{7} & S_{8} \\
0 & 0 & 0 & 0 & 0 & 0 & 0 & 0 & 1 & 0 & 0 & 0 \\
0 & 0 & 0 & 0 & 0 & 0 & 0 & 0 & 0 & 0 & -T_{8} & T_{7} \\
0 & 0 & 0 & 0 & 0 & 0 & 0 & 0 & -T_{6} & T_{5} & T_{6} & -T_{5} \\
S_{9} & S_{10} & S_{11} & S_{12} & S_{13} & S_{14} & S_{15} & S_{16} & S_{17} & S_{18} & -T_{5} & -T_{6}
\end{array}\right]
$$


where

$$
\begin{aligned}
S_{1}=G_{2}+N_{3} \bar{H}_{V} G_{1}, & S_{2}=G_{1}+N_{3} \bar{H}_{V} G_{2}, \\
S_{3}=-G_{6}-N_{3} \bar{H}_{V} G_{5}, & S_{4}=G_{5}-N_{3} \bar{H}_{V} G_{6}, \\
S_{5}=\frac{N_{34}}{\bar{H}_{V}} T_{5}, & S_{6}=\frac{N_{34}}{\bar{H}_{V}} T_{6}, \\
S_{7}=\frac{-N_{34}}{\bar{H}_{V}} T_{5}, & S_{8}=\frac{-N_{34}}{\bar{H}_{V}} T_{6}, \\
S_{9}=N_{12} \bar{H}_{V} G_{2}, & \mathrm{~S}_{10}=N_{12} \bar{H}_{V} G_{1}, \\
\mathrm{~S}_{11}=-N_{12} \bar{H}_{V} G_{6}, & \mathrm{~S}_{12}=N_{12} \bar{H}_{V} G_{5}, \\
\mathrm{~S}_{13}=-N_{12} \bar{H}_{V} G_{2}, & \mathrm{~S}_{14}=-N_{12} \bar{H}_{V} G_{1}, \\
\mathrm{~S}_{15}=N_{12} \bar{H}_{V} G_{6}, & \mathrm{~S}_{16}=-N_{12} \bar{H}_{V} G_{5}, \\
\mathrm{~S}_{17}=T_{5}-N_{1} \bar{H}_{S} T_{6}, & \mathrm{~S}_{18}=T_{6}+N_{1} \bar{H}_{S} T_{5}, \\
G_{1}=\operatorname{Cosh}\left(\bar{H}_{V} \alpha\right), & G_{2}=\operatorname{Sinh}\left(\bar{H}_{V} \alpha\right), \\
G_{3}=\operatorname{Cosh}\left(\bar{H}_{V}\right), & G_{4}=\operatorname{Sinh}\left(\bar{H}_{V}\right), \\
G_{5}=\operatorname{Cos}\left(\bar{H}_{V} \alpha\right), & G_{6}=\operatorname{Sin}\left(\bar{H}_{V} \alpha\right), \\
G_{7}=\operatorname{Cosh}\left(\bar{H}_{V}\right), & G_{8}=\operatorname{Sin}\left(\bar{H}_{V}\right), \\
T_{5}=\operatorname{Cos}\left(\bar{H}_{S} \alpha\right), & T_{6}=\operatorname{Sin}\left(\bar{H}_{S} \alpha\right), \\
T_{7}=\operatorname{Cos}\left(\bar{H}_{S}\right), & T_{8}=\operatorname{Sin}\left(\bar{H}_{S}\right), \\
N_{12}, & N_{34}=\frac{N_{3}}{N_{4}} .
\end{aligned}
$$

(1) Cantilever beam specimen

(6) Function generator

(2) Accelerometer

(7) Power supply

(3) Vibration analyzer

(8) Power amplifier

(4) Vibration indicator

(9) Vibration exciter

(5) Distribution box

FIGURE 3: Schematic block diagram of experimental setup for the cantilever beam.

TABle 1: Material properties of Aluminium-Alloy, 2014- $\mathrm{T}_{4}$.

\begin{tabular}{lccccc}
\hline $\begin{array}{l}\text { Young's } \\
\text { modulus, } E\end{array}$ & $\begin{array}{c}\text { Density, } \\
\rho\end{array}$ & $\begin{array}{c}\text { Poisson's } \\
\text { ratio, } \mu\end{array}$ & $\begin{array}{c}\text { Length, } \\
L\end{array}$ & $\begin{array}{c}\text { Width, } \\
W\end{array}$ & $\begin{array}{c}\text { Thickness, } \\
T\end{array}$ \\
\hline $72.4 \mathrm{GPa}$ & $2.8 \mathrm{gm} / \mathrm{cc}$ & 0.33 & $800 \mathrm{~mm}$ & $50 \mathrm{~mm}$ & $6 \mathrm{~mm}$ \\
\hline
\end{tabular}

depths varying from $1 \mathrm{~mm}$ to $5 \mathrm{~mm}$ by a step of $1 \mathrm{~mm}$. The cracks were prepared by fine saw cuts perpendicular to the longitudinal axis. This ensures that the crack remains open during the vibrations. At each step the first three bending natural frequencies of the cracked beams were measured. Table 1 gives the corresponding bending natural frequencies of the intact and cracked beams. These specimens are set to vibrate under the 1st, 2nd, and 3rd modes of vibrations and the corresponding amplitudes are recorded in the vibration indicator. Experimental results of frequencies of transverse vibration at various locations along the length of the beam are recorded by positioning the vibration pickup and tuning the vibration generator at the corresponding resonant frequencies.

DE was employed to detect cracks utilizing the results from the experimental study. A comparison was made between the experimentally measured natural frequencies of the damaged beam and the ones obtained through the cracked beam model using the objective function as discussed in Section 4.4.

The theoretical results are better than the experimental ones, because of measurement errors. The proposed methods and mode shapes for different crack locations (i.e., $200 \mathrm{~mm}$, $400 \mathrm{~mm}$, and $600 \mathrm{~mm}$ from the clamped end) and crack
Several tests are conducted using the experimental setup on aluminum beam specimens $(800 \times 50 \times 6 \mathrm{~mm})$ with a transverse crack for determining the natural frequencies 
have been applied to nine damage cases obtained by combining three different crack positions and three different crack depths.

Then theoretical and experimental results are compared by using the differential evolution method.

\section{Differential Evolution}

The differential evolution (DE) algorithm is a population based evolutionary algorithm developed by nondifferentiable continuous space functions [26]. Like the genetic algorithm, the optimization process in differential evolution is subject to three basic operations: mutation, crossover, and selection. $\mathrm{DE}$, at the start, randomly initializes a population of size NP solution vectors, which is evolved over $G$ generations to reach an optimal solution. The population size NP is kept constant during the minimization process. Each individual $X_{i}$ of the population is a vector that contains a set of $D$ optimization parameters as the problem decision variables and can be expressed as

$$
X_{i, G}=\left\{x_{i, G}^{1}, \ldots, x_{i, G}^{D}\right\}
$$

In the crack detection problem the search space is bounded by the parameters such as crack location and depth in the cantilever beam. The minimum and maximum parameter bounds are $X_{\min }=\left\{x_{\min }^{1}, \ldots, x_{\min }^{D}\right\}$ and $X_{\max }=$ $\left\{x_{\max }^{1}, \ldots, x_{\max }^{D}\right\}$, respectively. The initial value of the $j$ th parameter in the $i$ th individual at the iteration $G=0$ can be expressed as

$$
x_{i, 0}^{j}=x_{\min }^{j}+\operatorname{rand}\left(x_{\max }^{j}-x_{\min }^{j}\right),
$$

where rand represents a uniformly distributed random variable within the range $[0,1]$.

4.1. Mutation Operation. For each target vector $X_{i, G} i=$ $1,2, \ldots, N P$, a mutant vector is generated according to the following mutation strategy that is used in the present work:

$$
V_{i, G+1}=X_{i, G}+F \cdot\left(X_{\text {best }, G}-X_{i, G}\right)+F \cdot\left(X_{r 1, G}-X_{r 2, G}\right) \text {, }
$$

Where $r_{1}, r_{2} \in\{1,2, \ldots, N P\}$ are mutually different integer numbers and are also different from the index $i$.

The indices $r_{1}^{i}, r_{2}^{i}, r_{3}^{i}, r_{4}^{i}$, and $r_{5}^{i}$ are mutually exclusive integers randomly generated within the range $[1, \mathrm{NP}]$, which are also different from the index $i$. These indices are randomly generated once for each mutant vector. The scaling factor $F$ is a positive control parameter for scaling the difference vector.

4.2. Crossover Operation. The crossover operation is introduced in the DE algorithm, in order to increase the diversity of the vectors. The crossover operation is carried out by randomly exchanging between the original vectors of the population $X_{i, G}$ and those of the mutant population $V_{i, G+1}$ to obtain the trial vectors $Z_{i, G+1}=\left(z_{i, G+1}^{1}, \ldots, z_{i, G+1}^{D}\right)$. The trial vector is determined by a parameter called crossover probability $(\mathrm{CR} \in[0,1])$ as follows:

$$
z_{i, G}^{j}= \begin{cases}v_{i, G}^{j}, & \text { if }(\text { rand } \leq \mathrm{CR}) \text { or } j=j_{\text {rand }}, \\ x_{i, G}^{j}, & \text { otherwise }\end{cases}
$$

where $j_{\text {rand }}$ is a randomly chosen integer in the range $[1, D]$.

4.3. Selection. In order to decide if a vector $Z_{i}$ may be the element of the new population of generation $G+1$, each vector $z_{i, G+1}$ is compared to the previous corresponding target vector $x_{i, G}$. If vector $z_{i, G+1}$ yields a smaller objective function value than $x_{i, G}$, then $x_{i, G+1}$ is set to $z_{i, G+1}$; otherwise, the old value $x_{i, G}$ is retained. The selection operation can be expressed as

$$
X_{i, G+1}= \begin{cases}Z_{i, G}, & \text { if } f\left(Z_{i, G}\right) \leq f\left(X_{i, G}\right) \\ X_{i, G}, & \text { otherwise }\end{cases}
$$

with $i=1,2, \ldots$, NP.

The aforementioned steps are repeated generation after generation until some specific termination criteria are satisfied. The algorithmic description of $\mathrm{DE}$ is summarized in Table 1.

4.4. Objective Function Based on Vibration Data. Damage in a structure makes changes in vibrational parameters such as natural frequencies and mode shapes. In this current study natural frequencies are taken as the damage indicator as they are easier to measure than mode shapes. The objective function chosen for damage estimation is a minimization optimization problem. The objective function based on natural frequencies can be expressed as

$$
F=\sum_{i=1}^{n} \frac{1}{w_{i}}\left|\left(f_{i}^{c}-f_{i}^{m}\right)\right|,
$$

where $f_{i}^{m}$ and $f_{i}^{c}$ are the measured natural frequencies and natural frequencies obtained from a theoretical spring model based on the assumed crack location and depth, respectively. The $n$ is the number of natural frequencies used to evaluate the objective function and $w_{i}$ is a weighting factor, whose values are considered to be $i$. In the present work, the first three natural frequencies are considered to determine the objective function.

Stopping Rule. The iterative procedure is terminated when a predefined number of generations or a computational error of $10^{-4}$ is reached.

\section{Pseudo code for DE}

(1) Generate a population of solution vectors.

(2) Evaluate the best member of the population $X_{\text {best }, i}$.

(3) Carry out the mutation operation $V_{i, G+1}$.

(4) Perform the crossover strategy $Z_{i, G+1}$.

(5) Check the bound constraint.

(6) Do the selection. 
TABLE 2: Measured natural frequencies for single crack cantilever beam.

\begin{tabular}{lccccc}
\hline \multirow{2}{*}{ Sl. No. } & \multicolumn{2}{c}{ Crack } & \multicolumn{3}{c}{ Bending frequencies $(\mathrm{Hz})$} \\
& Location $(\mathrm{mm})$ & Depth $(\mathrm{mm})$ & $f_{1}$ & $f_{2}$ & $f_{3}$ \\
\hline 1 & No crack & 0.0 & 7.0683 & 44.2951 & 124.0278 \\
2 & 300 & 1.2 & 7.0589 & 44.2267 & 123.8194 \\
3 & 300 & 1.8 & 7.0466 & 44.1380 & 123.5518 \\
4 & 300 & 2.4 & 7.0265 & 43.9916 & 123.1154 \\
5 & 450 & 1.2 & 7.0654 & 44.2564 & 123.9431 \\
6 & 450 & 1.8 & 7.0617 & 44.0162 & 123.8339 \\
7 & 450 & 2.4 & 7.0556 & 43.7567 & 123.6551 \\
8 & 600 & 1.2 & 7.0678 & 44.2532 & 123.6751 \\
9 & 600 & 1.8 & 7.0673 & 44.1985 & 123.2185 \\
10 & 600 & 2.4 & 7.0665 & 44.1068 & 122.4656 \\
\hline
\end{tabular}

TABLE 3: Calculated frequencies using theoretical analysis.

\begin{tabular}{lccccc}
\hline \multirow{2}{*}{ Sl. No. } & \multicolumn{2}{c}{ Crack } & \multicolumn{3}{c}{ Bending frequencies $(\mathrm{Hz})$} \\
& Location $(\mathrm{mm})$ & Depth $(\mathrm{mm})$ & $f_{1}$ & $f_{2}$ & $f_{3}$ \\
\hline 1 & No crack & 0.0 & 7.6829 & 47.1839 & 132.1165 \\
2 & 200 & 1.8 & 7.4123 & 46.6920 & 130.2206 \\
3 & 400 & 1.8 & 7.3648 & 46.6467 & 130.7678 \\
4 & 600 & 1.8 & 7.4898 & 46.6006 & 130.5848 \\
5 & 200 & 2.4 & 7.3745 & 46.6821 & 130.3828 \\
6 & 400 & 2.4 & 7.3547 & 46.1335 & 130.7672 \\
7 & 600 & 2.4 & 7.2969 & 46.5039 & 129.1213 \\
\hline
\end{tabular}

TABLE 4: Crack detection results of the theoretical study by applying DE.

\begin{tabular}{|c|c|c|c|c|c|}
\hline \multicolumn{2}{|c|}{ Measured crack } & \multicolumn{4}{|c|}{ Predicted results $(\mathrm{mm})$ using $(\mathrm{DE})$} \\
\hline $\begin{array}{l}\text { Location } \\
(\mathrm{mm})\end{array}$ & $\begin{array}{l}\text { Depth } \\
(\mathrm{mm})\end{array}$ & $\begin{array}{l}\text { Location } \\
(\mathrm{mm})\end{array}$ & $\%$ err & $\begin{array}{l}\text { Depth } \\
(\mathrm{mm})\end{array}$ & $\%$ err \\
\hline 200 & 1.8 & 200.012 & 0.006 & 1.8002 & 0.011 \\
\hline 200 & 2.4 & 200.103 & 0.034 & 2.4006 & 0.025 \\
\hline 400 & 1.8 & 399.987 & 0.003 & 1.8000 & 0.000 \\
\hline 400 & 2.4 & 400.054 & 0.014 & 2.3964 & 0.151 \\
\hline 600 & 1.8 & 600.106 & 0.018 & 1.8006 & 0.034 \\
\hline 600 & 2.4 & 599.898 & 0.017 & 2.4005 & 0.021 \\
\hline
\end{tabular}

(7) Evaluate the best member of the population after selection $X_{\text {best, } i}$.

(8) If the stopping criterion is reached, then print the output results and stop; otherwise repeat Steps 2-7.

(9) Then go to Step 3.

\section{Results and Discussions}

The theoretical analysis and proposed DE algorithm were implemented using MATLAB 7.0 [27]. Based on the results obtained from the numerical, experimental, and proposed DE model, the following discussions can be made. A crack in a beam structure causes a change in the stiffness of the beam, which is in turn a function of crack location and
TABLE 5: Crack detection results of the experimental study by applying DE.

\begin{tabular}{|c|c|c|c|c|c|}
\hline \multicolumn{2}{|c|}{ Measured crack } & \multicolumn{4}{|c|}{ Predicted results (mm) using (DE) } \\
\hline $\begin{array}{l}\text { Location } \\
(\mathrm{mm})\end{array}$ & $\begin{array}{l}\text { Depth } \\
(\mathrm{mm})\end{array}$ & $\begin{array}{l}\text { Location } \\
(\mathrm{mm})\end{array}$ & $\%$ err & $\begin{array}{l}\text { Depth } \\
(\mathrm{mm})\end{array}$ & $\%$ err \\
\hline 300 & 1.8 & 265.14 & 11.62 & 1.961 & 8.94 \\
\hline 300 & 2.4 & 332.04 & 10.68 & 2.604 & 8.50 \\
\hline 450 & 1.8 & 399.96 & 11.12 & 1.633 & 8.72 \\
\hline 450 & 2.4 & 496.58 & 10.35 & 2.588 & 7.83 \\
\hline 600 & 1.8 & 537.66 & 10.39 & 1.940 & 7.78 \\
\hline 600 & 2.4 & 540.72 & 9.88 & 2.228 & 7.16 \\
\hline
\end{tabular}

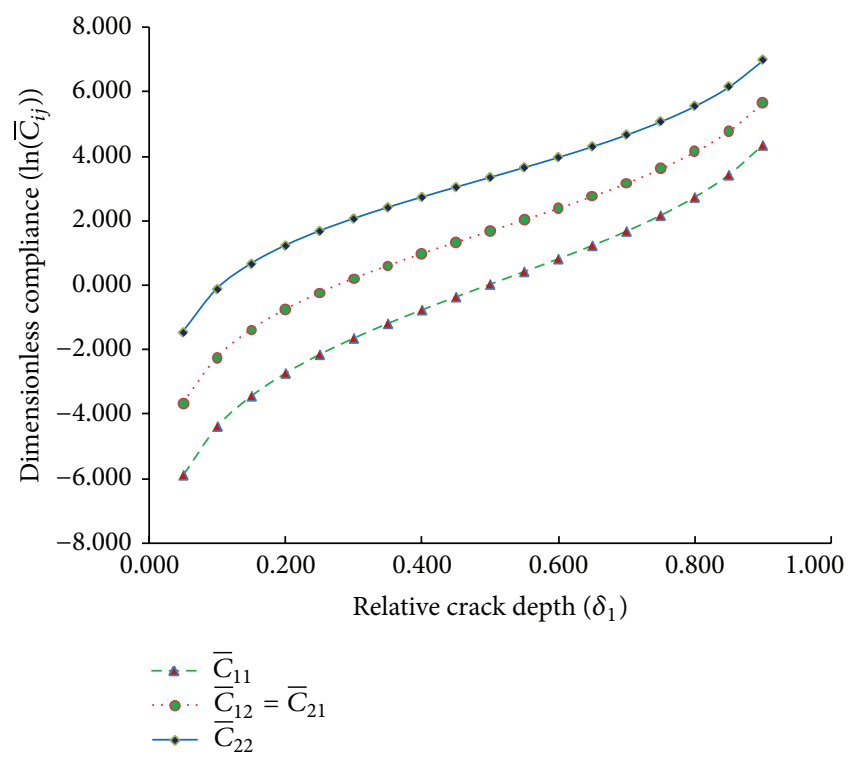

FIGURE 4: Dimensionless compliance $\left(\ln \overline{\left(C_{11}\right.}\right)$ versus relative crack depth $\left(\delta_{1}\right)$.

crack depth. As discussed in Section 2, the dimensionless compliance matrix is used to evaluate the stiffness matrix. The dimensionless compliances $\left(\bar{C}_{11}, \bar{C}_{12}=\bar{C}_{21}, \bar{C}_{22}\right)$ increasing with the increase in relative crack depth are as shown in Figure 4.

For crack location (e.g., $100 \mathrm{~mm}$ ) and relative crack depths $\left(\delta_{1}=b_{1} / T\right)$ (e.g., 0.1$)$, the first three mode shapes are presented graphically in Figure 5, where it is observed that there are reasonable changes in mode shapes due to the presence of a crack in the beam.

The numerical results for the relative amplitude of transverse vibration at different locations of aluminium alloy 2014- $T_{4}$ cracked specimens for the first three modes are obtained using the theoretical model as per (16a) to (16d) with the help of computer programming. These results for cracked and uncracked specimens are presented in Figure 5 for comparison. It can be seen that the deviations are more prominent with higher modes of vibrations. Table 1 presents the first three natural frequencies obtained from the theoretical model, which is subsequently used in the DE algorithm for crack identification. 


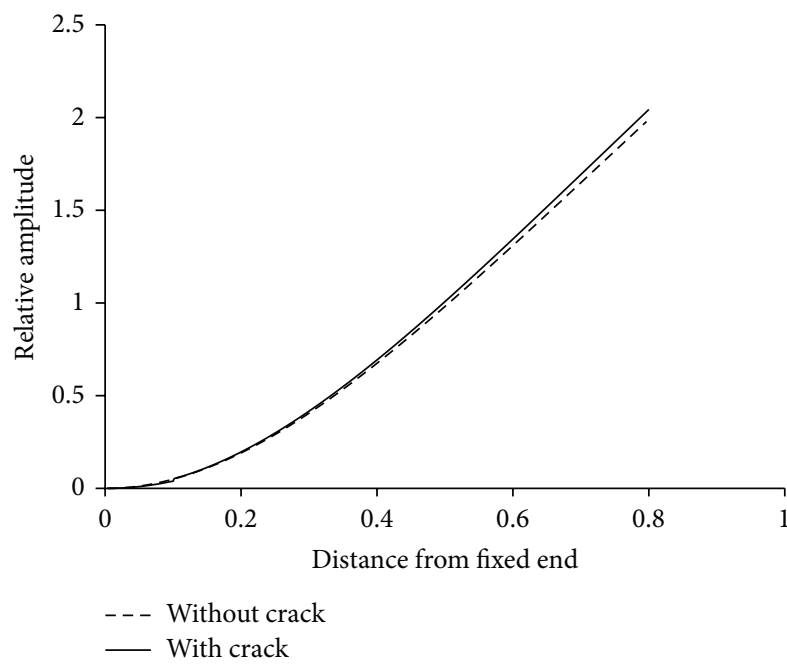

(a)

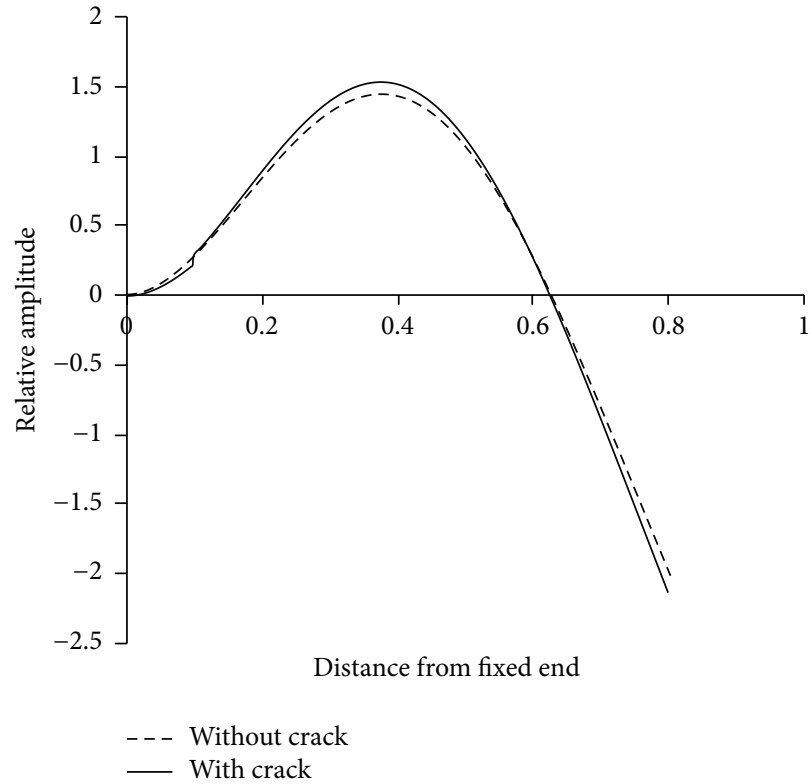

(c)

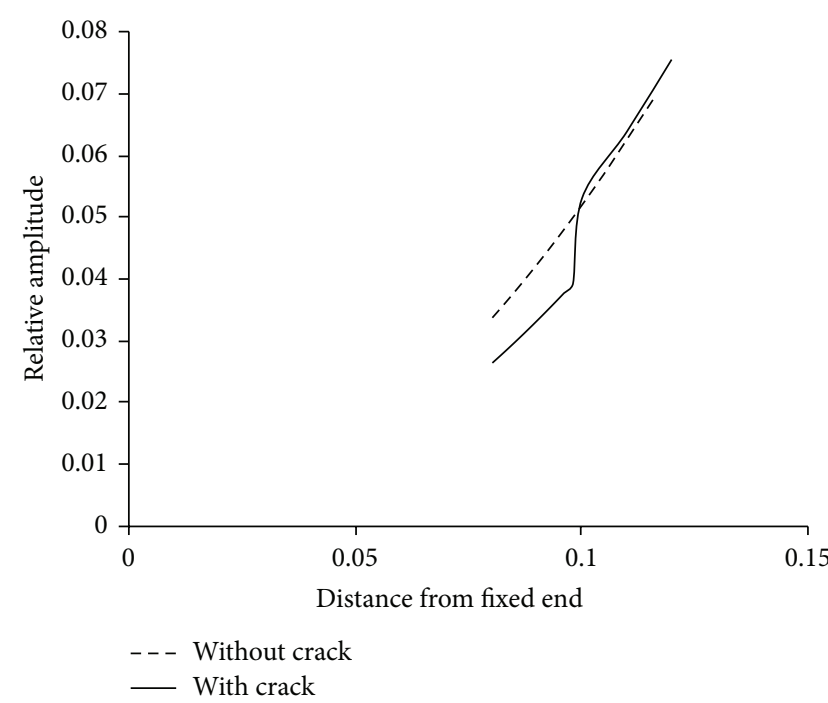

(b)

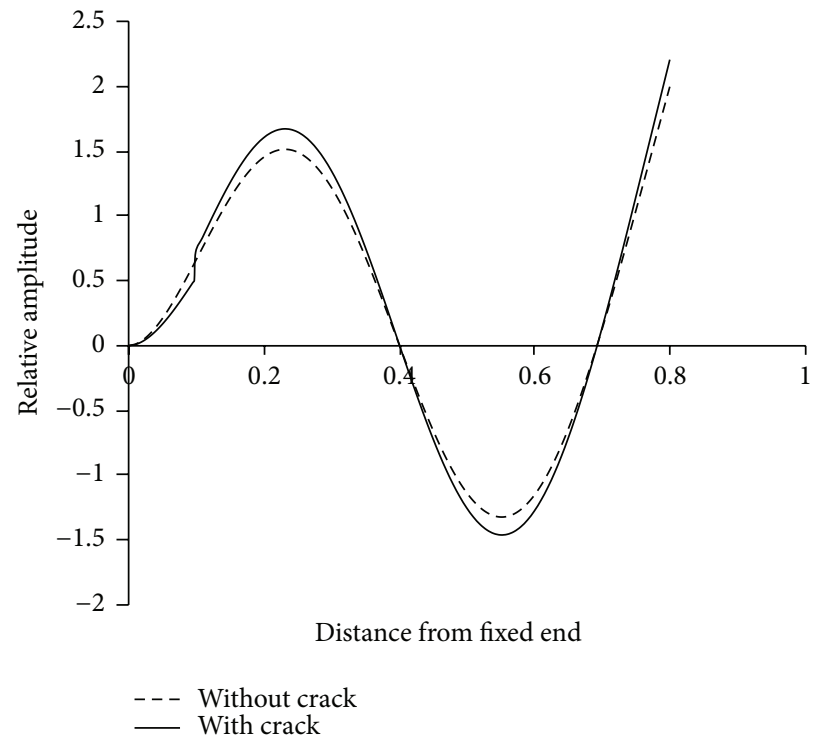

(d)

Figure 5: (a) Relative amplitude versus distance from the fixed end (1st mode of vibration), $L_{c}=100 \mathrm{~mm}, b_{1} / T=0.1$. (b) Magnified view of Figure 4(a) at the location. (c) Relative amplitude versus distance from the fixed end (2nd mode of vibration), $L_{c}=100 \mathrm{~mm}, b_{1} / T=0.1$. (d) Relative amplitude versus distance from the fixed end (3rd mode of vibration), $L_{c}=100 \mathrm{~mm}, b_{1} / T=0.1$.

5.1. Computational Results/Simulation Results. Different crack conditions have been taken to evaluate the performance of the proposed DE. Simulation results of 4 test points have been presented in this paper.

Figure 6 shows the convergence trend of the fitness value of the objective function with the number of iterations. Figures $6(a)-6(c)$ show the convergence trend for the theoretical model and Figure 6(d) is for the experimental study. As it can be seen from Figures 6(a)-6(c), the number of iterations for convergence increases with the increase in crack location for the same crack depth. Figure 6(d) shows that the fitness value does not converge for the predefined number of iterations.
5.2. Simulation Results. Different crack conditions have been taken to evaluate the performance of DE. Simulation results of 6 test points have been presented in this paper. The error is calculated using the following formula:

$$
\text { \%error }=\left|\frac{\text { predicted value }- \text { actual value }}{\text { actual value }}\right| \times 100 \text {. }
$$

Table 4 represents the validation of the proposed DE model with the results obtained as per the theoretical model. The percentage deviations in the results obtained by the experimental study and DE model are presented in Table 5. 


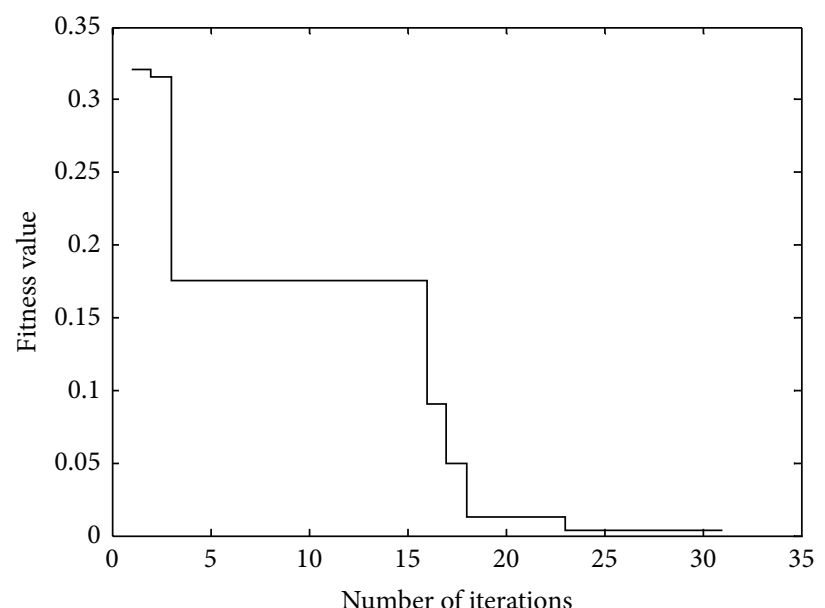

(a)

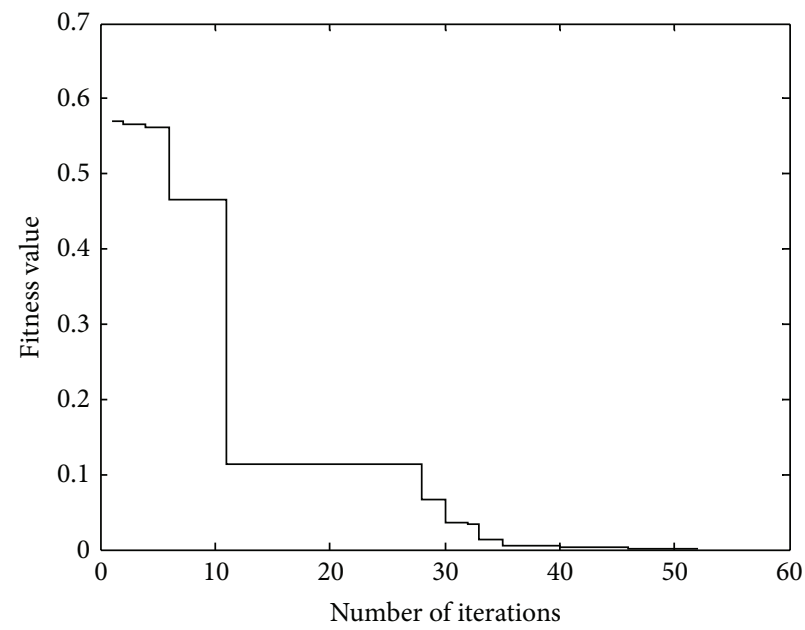

(c)

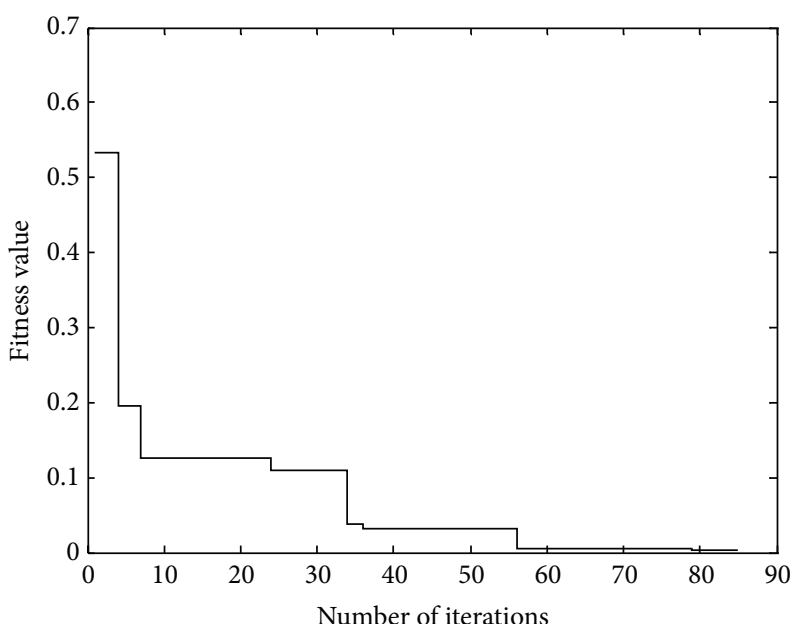

(b)

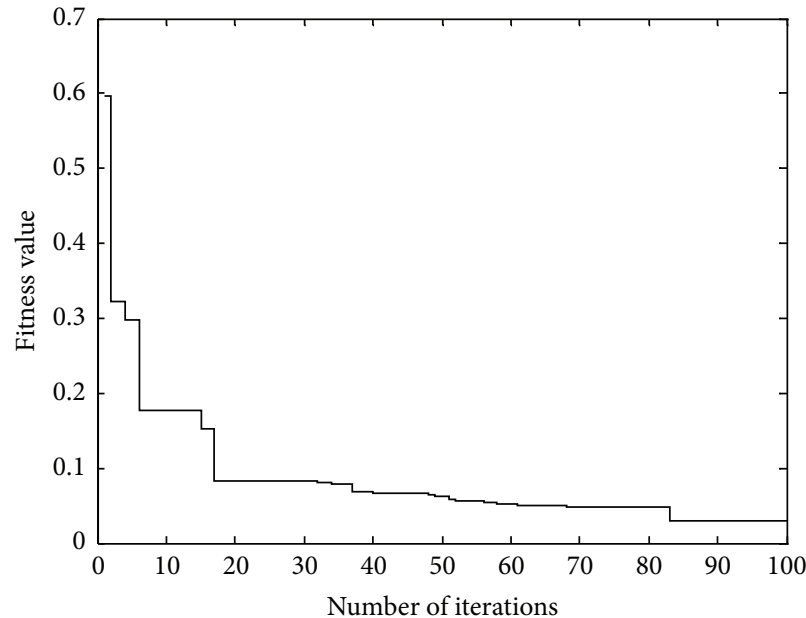

(d)

Figure 6: (a) Loc $=200$, depth $=2.4$. (b) Loc $=600$, depth $=2.4$. (c) $\operatorname{Loc}=400$, depth $=2.4$. (d) Loc $=300$, depth $=2.4$.

Table 2 shows the first three natural frequencies of the cracked cantilever beam at different crack locations and depths. The theoretical natural frequencies of the test specimens are presented in Table 3. It is found that the estimation error is reduced by increasing the crack depths for the same locations (Table 5). Also by increasing the distance of the crack from the fixed end of the beam, the estimation error has been found to be decreasing for similar crack depth. Tables 4 and 5 represent the validation of the proposed DE model with the results obtained through the theoretical and experimental models, respectively.

\section{Conclusions}

Based on the results and discussions thereof, the following conclusions can be made. The mode shapes and bending frequencies of the cracked elastic structures are strongly influenced by the crack location and its intensity. Though significant changes in mode shapes are observed in the vicinity of crack location, these deviations in mode shapes cannot be used as a measuring tool in the prediction of crack location and its intensity. In the present work, the proposed DE algorithm is found to be an efficient method for damage quantification in terms of crack location and crack depth by minimizing the error between measured and predicted frequencies. It was also observed that the error associated with the prediction is less in the theoretical model as compared to the experimental model. The present work can be implemented for damage assessment in different structures.

\section{References}

[1] P. Cawley and R. D. Adams, "Location of defects in structures from measurements of natural frequencies," Journal of Strain Analysis for Engineering Design, vol. 14, no. 2, pp. 49-57, 1979.

[2] A. D. Dimarogonas, "Vibration of cracked structures: a state of the art review," Engineering Fracture Mechanics, vol. 55, no. 5, pp. 831-857, 1996. 
[3] S. W. Doebling, C. F. Farrar, M. B. Prime, and D. W. Shevits, "Damage identification and health monitoring of structural and mechanical systems from changes in their vibration characteristics: a literature review," Los Alamos National Laboratory, Los Alamos, NM, USA, 1996.

[4] C. Boller, "Next generation structural health monitoring and its integration into aircraft design," International Journal of Systems Science, vol. 31, no. 11, pp. 1333-1349, 2000.

[5] D. Maity and R. R. Tripathy, "Damage assessment of structures from changes in natural frequencies using genetic algorithm," Structural Engineering and Mechanics, vol. 19, no. 1, pp. 21-42, 2005.

[6] W. Fan and P. Qiao, "Vibration-based damage identification methods: a review and comparative study," Structural Health Monitoring, vol. 10, no. 1, pp. 83-111, 2011.

[7] D. N. Thatoi, H. C. Das, Parhi, and D. R. :, "Review of techniques for fault diagnosis in damaged structure and engineering system," Advances in Mechanical Engineering, vol. 2012, article 32756, 11 pages, 2012.

[8] A. Sengupta and A. Upadhyay, "Locating the critical failure surface in a slope stability analysis by genetic algorithm," Applied Soft Computing Journal, vol. 9, no. 1, pp. 387-392, 2009.

[9] R. Perera, A. Ruiz, and C. Manzano, "An evolutionary multiobjective framework for structural damage localization and quantification," Engineering Structures, vol. 29, no. 10, pp. 25402550, 2007.

[10] X. Fang, H. Luo, and J. Tang, "Structural damage detection using neural network with learning rate improvement," Computers and Structures, vol. 83, no. 25-26, pp. 2150-2161, 2005.

[11] K. M. Saridakis, A. C. Chasalevris, C. A. Papadopoulos, and A. J. Dentsoras, "Applying neural networks, genetic algorithms and fuzzy logic for the identification of cracks in shafts by using coupled response measurements," Computers and Structures, vol. 86, no. 11-12, pp. 1318-1338, 2008.

[12] Y. M. Kim, C. K. Kim, and G. H. Hong, "Fuzzy set based crack diagnosis system for reinforced concrete structures," Computers and Structures, vol. 85, no. 23-24, pp. 1828-1844, 2007.

[13] P. Beena and R. Ganguli, "Structural damage detection using fuzzy cognitive maps and Hebbian learning," Applied Soft Computing, vol. 11, no. 1, pp. 1014-1020, 2011.

[14] F. Kang, J. J. Li, and Q. Xu, "Damage detection based on improved particle swarm optimization using vibration data," Applied Soft Computing, vol. 12, no. 8, pp. 2329-2335, 2012.

[15] B. Samanta and C. Nataraj, "Use of particle swarm optimization for machinery fault detection," Engineering Applications of Artificial Intelligence, vol. 22, no. 2, pp. 308-316, 2009.

[16] M. Clerc, "The swarm and the queen: towards a deterministic and adaptive particle swarm optimization," in Proceeding of the International Coference on Entertainment Computing, pp. 19511957, Washington, DC, USA, I999.

[17] M. Marinaki, Y. Marinakis, and G. E. Stavroulakis, "Fuzzy control optimized by PSO for vibration suppression of beams," Control Engineering Practice, vol. 18, no. 6, pp. 618-629, 2010.

[18] O. Begambre and J. E. Laier, "A hybrid Particle Swarm Optimization-Simplex algorithm (PSOS) for structural damage identification," Advances in Engineering Software, vol. 40, no. 9, pp. 883-891, 2009.

[19] M. T. V. Baghmisheh, M. Peimani, M. H. Sadeghi, M. M. Ettefagh, and A. F. Tabrizi, "A hybrid particle swarm-Neldermead optimization method for crack detection in cantilever beams," Applied Soft Computing, vol. 12, no. 8, pp. 2217-2226, 2012.
[20] P. M. Pawar and R. Ganguli, "Genetic fuzzy system for damage detection in beams and helicopter rotor blades," Computer Methods in Applied Mechanics and Engineering, vol. 192, no. 1618, pp. 2031-2057, 2003.

[21] M. R. A. Rama, K. Lakshmi, and D. Venkatachalam :, "Damage diagnostic technique for structural health monitoring using POD and self adaptive differential evolution algorithm," Computers and Structures, vol. 106-107, pp. 228-244, 2012.

[22] T. W. Liao, “Two hybrid differential evolution algorithms for engineering design optimization," Applied Soft Computing, vol. 10, no. 4, pp. 1188-1199, 2010.

[23] S. Moradi and V. Tavaf, "Crack detection in circular cylindrical shells using differential quadrature method," International Journal of Pressure Vessels and Piping, 2013.

[24] L. Vincenzi, G. De Roeck, and M. Savoia, "Comparison between coupled local minimizers method and differential evolution algorithm in dynamic damage detection problems," Advances in Engineering Software, vol. 65, pp. 90-100, 2013.

[25] H. Tada, P. C. Paris, and G. R. Irwin, The Stress Analysis of Cracks Hand Book, Hellertown, Pa, USA, 1973.

[26] R. Storn and K. Price, "Differential evolution-a simple and efficient heuristic for global optimization over continuous spaces," Journal of Global Optimization, vol. 11, no. 4, pp. 341359, 1997.

[27] The Mathworks, MATLAB. 7.0.1.24704. 

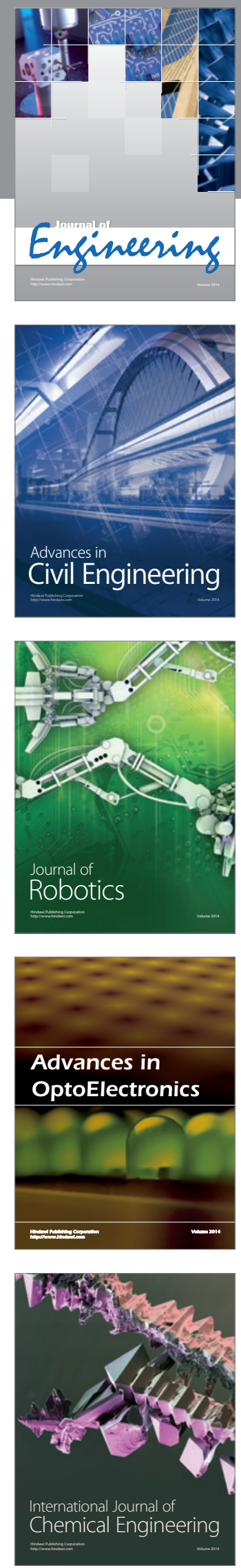

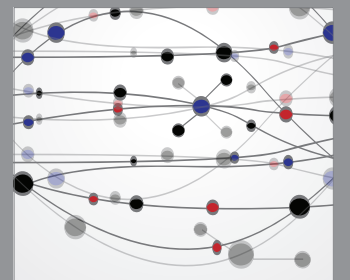

The Scientific World Journal
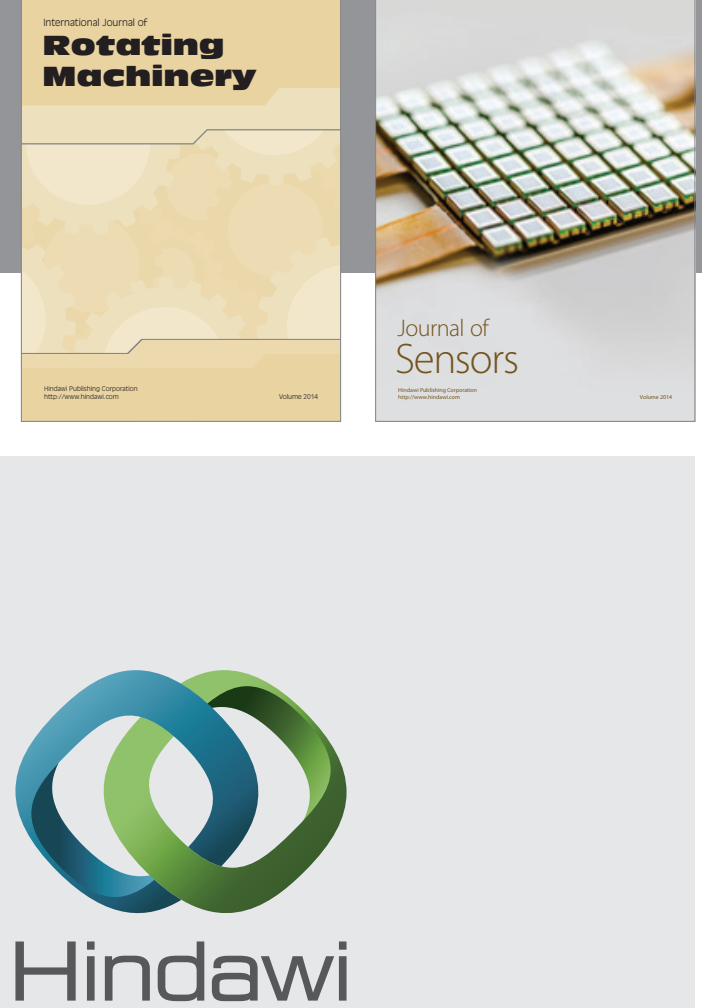

Submit your manuscripts at http://www.hindawi.com
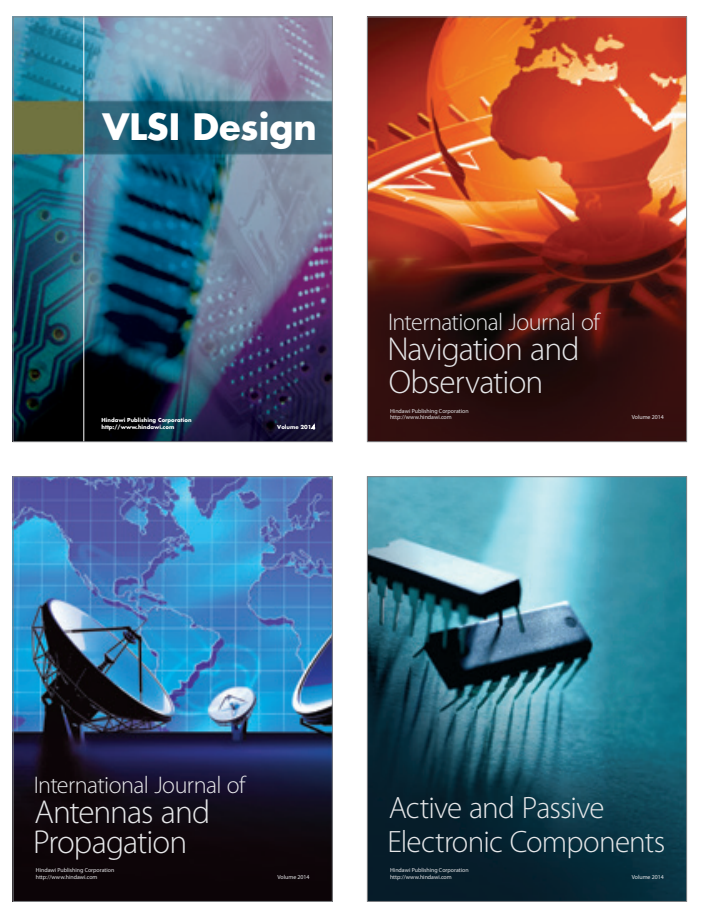
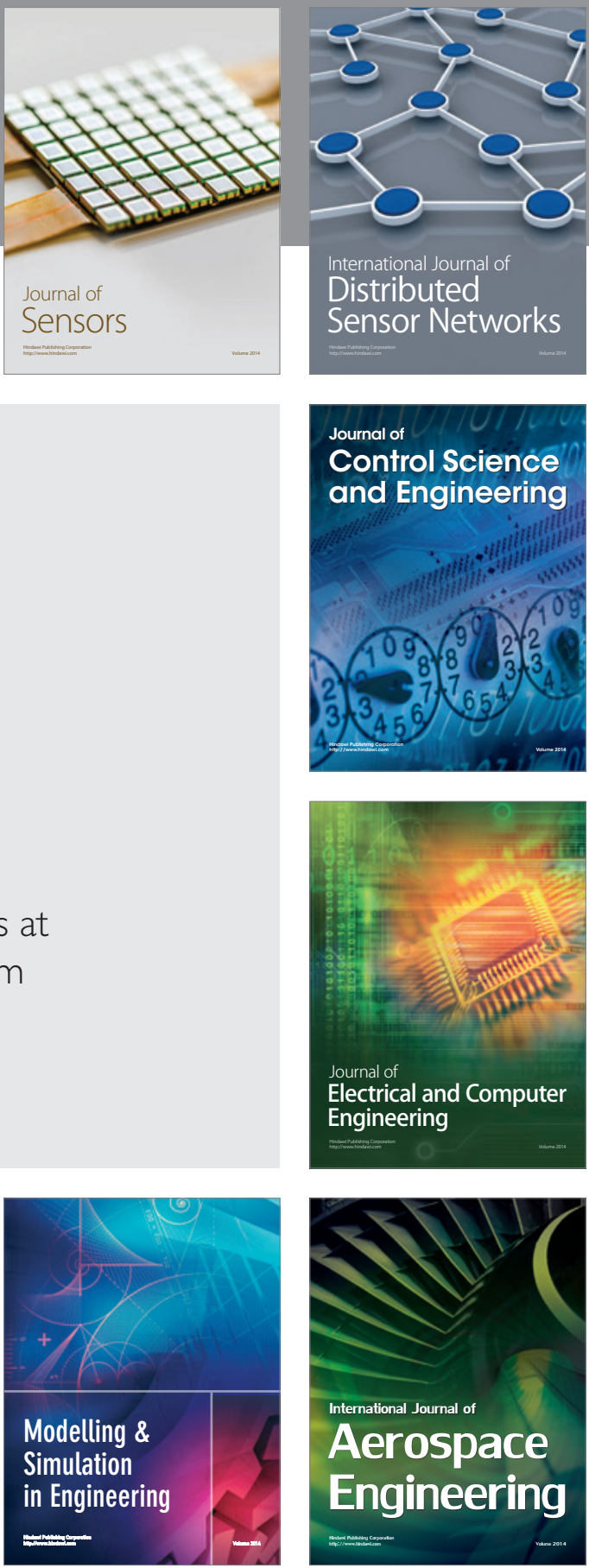

Journal of

Control Science

and Engineering
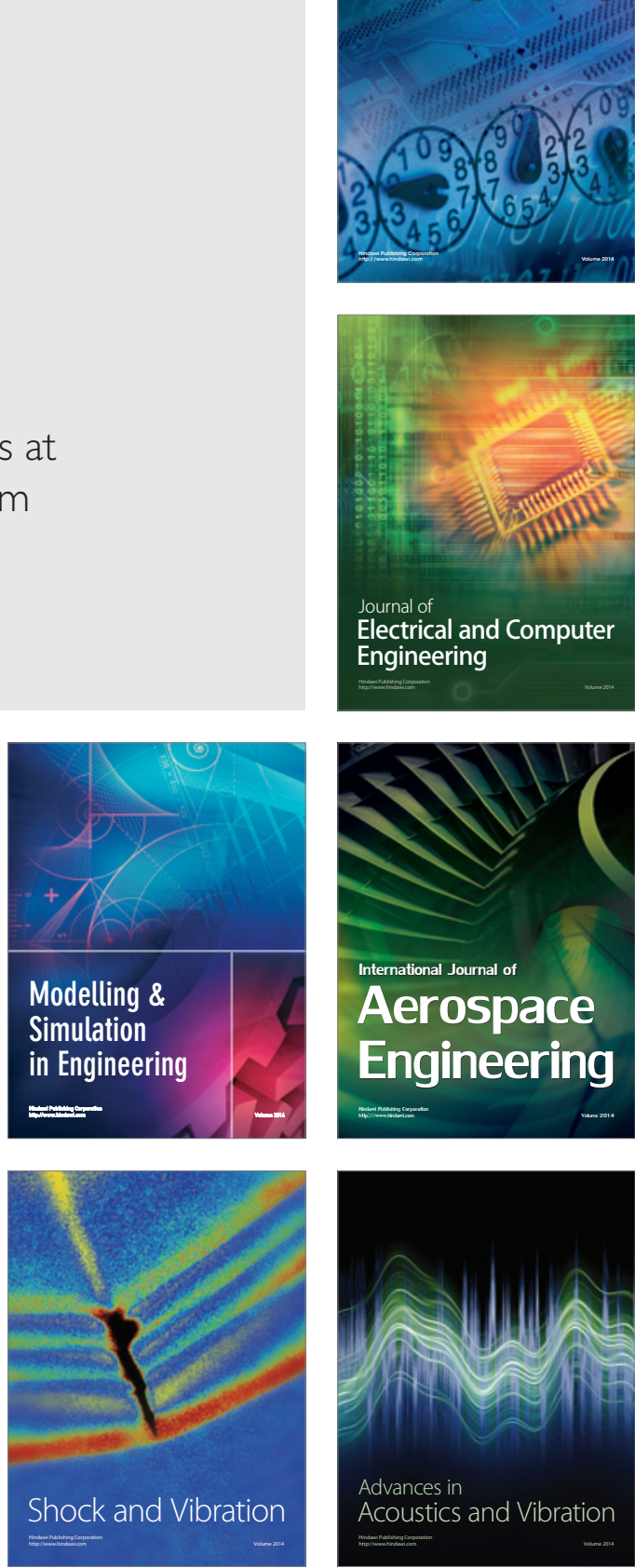